\title{
THE SEARCH FOR NEW AXIOMS IN THE HYPERUNIVERSE PROGRAMME
}

\author{
SY-DAVID FRIEDMAN*, CLAUDIO TERNULLO ${ }^{\dagger}$
}

\begin{abstract}
The Hyperuniverse Programme, introduced in Arrigoni and Friedman (2013), fosters the search for new set-theoretic axioms. In this paper, we present the procedure envisaged by the programme to find new axioms and the conceptual framework behind it. The procedure comes in several steps. Intrinsically motivated axioms are those statements which are suggested by the standard concept of set, i.e. the 'maximal iterative concept', and the programme identifies higher-order statements motivated by the maximal iterative concept. The satisfaction of these statements (HI-axioms) in countable transitive models, the collection of which constitutes the 'hyperuniverse' $(\mathbb{H})$, has remarkable first-order consequences, some of which we review in $\S 5$.
\end{abstract}

Keywords: new axioms, set-theoretic multiverse, Hyperuniverse Programme.

\section{NEW SET-THEORETIC AXIOMS}

Over the last years, there has been an intense debate within the set-theoretic community concerning the acceptance or non-acceptance of several set-theoretic statements such as $\mathrm{V}=\mathrm{L}$, large cardinals, axioms of determinacy $\left(\mathrm{AD}, \mathrm{PD}, \mathrm{AD}^{L(\mathbb{R})}\right)$ or forcing axioms (MA, PFA, etc.) and the discussion seems to be nowhere near being settled.

The received view concerning an axiom is that it should be 'self-evident', i.e., that it should be immediately, and with little effort, acknowledged as true. If such a view is still to be held, then there is no hope to accept the aforementioned statements as new axioms. ${ }^{1}$

But even if one discards the 'self-evidence view' as inapplicable, there are still deep issues which have to be addressed by anyone supporting the acceptance of one or more of the statements mentioned above and, more generally, of any axiom candidate.

First of all, there is often a lack of intrinsic motivation for such statements, where, by 'intrinsic', as explained at length in the sections below, we mean 'required by the concept

\footnotetext{
Date: October 28, 2015.

${ }^{*}$ Kurt Gödel Research Center for Mathematical Logic, Vienna. $\quad$ +431427750501 $\square$ sdf@logic.univie.ac.at.

${ }^{\dagger}$ Kurt Gödel Research Center for Mathematical Logic, Vienna. $\mathbb{\boldsymbol { \sigma }}+4368181536441 \square$ claudio.ternullo@univie.ac.at.

${ }^{1}$ Of course, it is also as much debatable that the standard axioms of set theory, that is, ZFC, are all 'self-evident'. A very natural case in point is the Axiom of Choice, but one may have equally reasonable reservations on the Axioms of Infinity, Replacement or Foundation. A thorough discussion of some of these issues can be found in Wang (1974), Maddy (1988a), Maddy (1988b) and Potter (2004).
} 
of set'. Secondly, the view that a new axiom should be accepted as true of the realm of sets has been seriously challenged by the independence phenomenon and the related existence of a set-theoretic multiverse: it is often relatively easy to produce a universe of sets which contradicts a given set-theoretic statement. Finally, all new axiom candidates are firstorder and one main worry we want to bring out in this paper is precisely that first-order principles may be too weak to capture further properties of the cumulative set-theoretic hierarchy.

One clear preliminary upshot of the informal considerations above is the following: it is unlikely that any new first-order axiom candidate will be accepted on its own as an intrinsically motivated principle of set theory. Granted, it might still be accepted on purely extrinsic grounds, but it is not clear that this would be sufficient evidence for its acceptance.

In this paper, we are going to propose an alternative way to identify new intrinsically motivated set-theoretic axioms, which originates from the conceptual framework of the Hyperuniverse Programme, as detailed in Arrigoni and Friedman (2013), and which fosters a revisionary conception of what a 'new' axiom is. In our view, new axioms are higherorder set-theoretic principles, more specifically principles expressing the maximality of the universe of sets $(V)$. The latter are strong mathematical propositions, some of which have been gradually isolated and examined in recent years in work by the first author and others, and, more recently, by the first author and Honzik. ${ }^{2}$ We believe that there is a sense in which such propositions, as will be presented in $\S 5$, can legitimately claim to be motivated by the concept of set and, by virtue of this, be viewed as intrinsically motivated new axioms.

It should be mentioned that all of these statements have striking first-order set-theoretic consequences, which we will describe in more detail in the next sections and this fact, although not representing an intrinsic justification for their acceptance, indisputably adds to their mathematical attractiveness.

One further goal of the Hyperuniverse Programme is to find one single 'optimal' maximality principle, whose acceptance would, thus, lead to identifying one single collection of first-order consequences. Therefore, our foundational project fosters the view that the procedure described here might also count as a procedure to find solutions to the open problems of set theory. However, the notion of 'solution', here, is inevitably as much revisionary as that of 'new' axiom.

The structure of the paper is as follows. In $\S 2-3$, we briefly discuss the features of 'intrinsic' evidence and set forth our conception of the set-theoretic universe as being a 'vertical' multiverse. In $\S 4$, we introduce the hyperuniverse as our auxiliary multiverse, wherein one can investigate the consequences of the maximality of $V$, through the use of $V$-logic. In $\S 5$, we enunciate maximality principles for $V$ which, in our view, are motivated by the concept of set. In $\S 6$ we discuss the notion of 'new' axiom qua $\mathbb{H}$-axiom, then, in $\S 7$, we proceed to make some final considerations. Finally, in $\S 8$, as an appendix, we present

\footnotetext{
${ }^{2}$ See, in particular, Friedman (2006), Arrigoni and Friedman (2012), Arrigoni and Friedman (2013) and Friedman and Honzik (nd).
} 
some results which show that a heavily investigated collection of new set-theoretic axioms, absoluteness axioms, which has recently received a lot of extrinsic support, may fall short of the requirements described above.

\section{INTRINSIC EVIDENCE FOR NEW AXIOMS}

2.1. Brief remarks on ontology and truth. In the next pages, we will be making frequent reference to issues of ontology and truth and it is maybe appropriate to briefly address these issues before examining the notion of intrinsic evidence.

On the grounds of what the programme aims to yield, i.e. new set-theoretic axioms, it is entirely natural to ask whether new axioms should be seen as 'true' statements of set theory, and in what sense. We will make it clear in the next section in what sense they should be viewed as 'true', but first we want to say something more general about 'truth'.

The programme's position is that axioms do not reflect truth in an independent realm of mathematical entities. It is rather the concept of set that plays a key role in our foundational project. As we shall see in the next subsection, and as is commonly acknowledged in set theory, the concept of set is instantiated by a specific mathematical structure, the cumulative hierarchy, but it does not automatically provide us with a fully determinate collection of properties of sets in this structure. Now, we believe that it is possible to derive properties of the concept of set which provide us with an indication of what further properties the set-theoretic hierarchy should have.

There is possibly a hint of realism in this position, insofar as we view the concept of set as being a 'stable' feature of our experience of sets and we subscribe to its stability in the sense that we do not question the ZFC axioms which are true of it.

However our view follows an overall epistemological concern, that of securing the truth of new axioms and of their first-order consequences through setting forth an alternative evidential framework for them which does not imply a pre-formed ontological picture. Therefore, ontology, in the most robust sense of the word, does not play a pre-eminent role in our project.

If there is a detectable ontological framework within our account, that is the core structure we identify in $\S 3$, i.e. the tower-like multiverse of $V_{\kappa}$ 's, where $\kappa$ is a strongly inaccessible cardinal. In turn, properties of this multiverse will motivate the adoption of one further ontological construct, the hyperuniverse, which consists of all countable transitive models. Neither multiverse is given a priori.

The maximality principles we will be concerned with quantify over extensions of $V$. However, our language is that of first-order ZFC, therefore maximality principles do not formally involve talk of classes. So, in the end, we have sets, and nothing else.

This ontological view might be seen as entailing a conception of truth that lacks the requisite strength to see axioms as 'true'. But in fact, as we will see, the concept of set is adequate to make strong claims about set-theoretic maximality, for instance alternative conceptions of vertical maximality are ruled out as unwarranted on the grounds of the concept itself. It is true, however, that in order to have models where new axioms 'live' one has to shift to countable transitive models and, thus, to a different framework of truth. 
But this is not overall necessary. One can still appreciate the force of maximality principles within the whole $V$ and, thus, stick to a vision of truth and ontology entirely befitting the concept of set. Further details on our positions will be given in the next few sections.

2.2. Two sources of evidence. Although the distinction is not entirely perspicuous, since Gödel (1947), it has become fairly commonplace in the literature to refer to two main different forms of evidence for the acceptance of an axiom as 'intrinsic' (internal) and 'extrinsic' (external) evidence. Very roughly, the distinction can be glossed as follows. Intrinsic evidence for an axiom is that following from the concept of set, whereas extrinsic evidence relates to the fruitfulness and success of an axiom, possibly also outside set theory. In other terms, an axiom may be accepted either because it expresses a 'necessary' property of sets or because it is corroborated by good results (and interesting practice) or for both reasons.

The issue of whether this distinction has any plausibility is beyond the scope of this article and, for the sake of our arguments, we will not challenge it. However, it should be noticed that, in our opinion, in opposition to the point of view expressed by some authors, 'intrinsicness' does not imply the view we have just informally rejected, that axioms should be 'self-evident'. ${ }^{3}$ In fact, an axiom may be true of the concept of set and not be immediately graspable as true. This is because not all true properties of the concept of set are immediately graspable. Therefore, arguably, it is our task to gradually uncover such properties, by clarifying the content of our intuitions. Ideally, we should be able to determine the properties of the concept of set, and possibly of other properties of clear set-theoretic relevance, by following what Potter calls the 'intuitive' method:

The intuitive method invites us instead to clarify our understanding of the concepts involved to such an extent as to determine (some of) the axioms they satisfy. The aim should be to reach sufficient clarity that we become confident in the truth of these axioms and hence, but only derivatively, in their consistency. If the intuitive method is successful, then, it holds out the prospect of giving us greater confidence in the truth of our theorems than the regressive method. ${ }^{4}$

But just what form of intuition does the intuitive method presuppose? Is intuition alone sufficient to justify the adoption of set-theoretic axioms? These are no doubt vexing questions on which we cannot fully dwell in this paper. However, some considerations are in order.

Intuition is, sometimes, construed in the Gödelian sense, as a faculty of perception which provides us with detailed information on mathematical objects. However, as we have seen,

\footnotetext{
${ }^{3}$ For instance, the equating of 'intrinsicness' with 'self-evidence' is clearly hinted at in the following passage of Maddy (1988a), p. 482: 'The suggestion is that the axioms of ZFC follow directly from the concept of set, that they are somehow 'intrinsic' to it (obvious, self-evident) [...]'.

${ }^{4}$ The 'regressive', as opposed to 'intuitive', method mentioned by Potter holds that '...the object of a good axiomatization is to retain as many as possible of the naive set-theoretic arguments which we remember with nostalgia from our days in Cantor's paradise, but to stop just short of permitting those arguments which lead to paradox.' (Potter (2004), p. 36)
} 
the programme does not commit itself to any form of object-realism. Therefore, our appeal to intuition and the intuitive method should be construed in the following way: as hinted at by Potter, we seem to have the ability to single out the relevant concepts and properties that are derivable from the concept of set.

As we shall see, the cumulative hierarchy instantiates the concept of set (as described below) and its maximality seems to follow naturally. Now, does that mean that we need to have access to platonistic entities in order to successfully carry out this task? We do not have a definite answer to this question, but, on the grounds of the considerations made in the previous section, it seems natural to tentatively rule out such possibility: realism should not extend so far as to postulate the existence of an independent, pre-formed ontology, but rather only postulate a stable concept of set, from which further properties of sets can be derived.

It should be noticed that we do not hold that our maximality principles, such as the IMH, become thus straightforwardly 'intrinsically justified'. What we believe to be intrinsically justified by the concept of set is rather the feature of the maximality of the cumulative hierarchy and, consequently, its maximal extendibility. Maximality principles take different forms, so we could, at most, say that such forms are intrinsically motivated, insofar as maximality, in general, is an intrinsically justified feature of the concept of set.

There is, of course, further work to be done to establish the stronger claim that some of our maximality principles are intrinsically justified, and we can only hope that further intrinsic evidence may, one day, substantially help bolster this claim.

2.3. The maximum iterative concept. Although it is not clear what Cantor took 'sets' to be at the beginning of his set-theoretic investigations, over the years increasingly wide agreement has been reached that the concept implies an account of the iterative formation of all sets along stages indexed by the ordinals. By this account, each set belongs to a stage of what has been called the cumulative hierarchy, starting with the empty set, and then iterating the power-set at all successor stages and the union of all sets formed in previous stages at limit stages of the hierarchy.

In fact, the iterative conception is more correctly referred to as the 'maximal iterative conception':

[MIC] 1) All sets which can be formed at each stage are actually formed. 2) The formation of sets should continue as far as possible.

The vocabulary used in the formulation of the $[\mathrm{MIC}]$ has been variously interpreted as hiding temporal, modal and, in general, metaphysical forms of mutual dependency among sets, elements and stages, and this aspect is responsible for some sort of conceptual opacity in the $[\mathrm{MIC}]^{5}$

However, leaving aside such troubles for the time being, it seems clear that the basic rationale underlying the $[\mathrm{MIC}]$ is that the procedures to form sets ought not to be constrained

\footnotetext{
${ }^{5}$ For an exhaustive overview of these issues see, again, Potter (2004), in particular, pp. 34-41, or Jané (2005b).
} 
by 'internal' limitations, that is, by mathematical principles hindering the maximisation and the continuation of such formation. This line of thought has been distinctly referred to in a fortunate article by Bernays as 'quasi-combinatorialism', the conceptual attitude which would allow one to treat and manipulate all mathematical objects, both finite and infinite, and combinations thereof, as fully determinate objects of thought. ${ }^{6}$ The maximal character of the [MIC], therefore, can be motivated using a 'quasi-combinatorial' conceptual framework, as, by this, one does not put any constraint on the class of producible sets.

These are well-known facts. Now, we want to take a step further. The intuitive method invites us to focus our attention on one specific feature of the [MIC] that we are going to use extensively in the rest of the paper. Suppose one takes the cumulative hierarchy to be a determined object of thought, $V$, the universe of sets. Then the [MIC] may also imply one further principle of 'plenitude', which can be formulated in the following way:

[MaxExt] Given a universe of sets, all possible extensions of it which can be formed are actually formed.

The 'extensions' referred to in [MaxExt] are given by the creation of 'new' sets in the only two ways we know, either adding new stages to the hierarchy or 'producing' new subsets at successor stages; thus the principle seems to be perfectly justified in light of the $[\mathrm{MIC}]$. However, the principle seems to shed light on one further dimension in the iteration, insofar as it assumes that the latter should get us beyond the universe itself. This automatically introduces the issue of whether we have grounds to believe that the universe is a determinate (actual) object of thought. As we shall see, there is, in fact, a way to interpret [MaxExt] in a way which remains faithful to its nature, but does not imply this kind of actualism.

In any case, our goal, for the time being, is to re-state the notion of 'intrinsicness' in the following way: intrinsic evidence for the acceptance of an axiom is that related to the [MIC], which, in particular, implies [MaxExt] as one of its features.

\section{Conceptions of $V$. The 'Vertical' multiverse}

3.1. What is $V$ ? The actualism/potentialism dichotomy. [MaxExt] seems to imply that the universe can be 'extended' and that there is no limitation on how much it can be extended. Extensions of the universe, as we said, are given by further stages in the cumulative hierarchy or new subsets. However, as anticipated, there is a difficulty in this point of view: the literal sense of the notion of 'extension', implies, at the very least, that what is extended is an object with 'boundaries', that is, a 'delimited' object. Now, it is not clear that the cumulative hierarchy is one such object. As a matter of fact, in the [MIC] there is nothing which commits us to seeing $V$ as a delimited object. On the contrary, it would seem that $V$ is best construed as an open-ended sequence of stages. On the other

\footnotetext{
6'In Bernays' own words, 'quasi-combinatorialism' ultimately refers to '...an analogy of the infinite with the finite.' See Bernays (1935), reprinted in Benacerraf and Putnam (1983), p. 259.
} 
hand, the standard interpretation of the first-order quantifiers is that they range over the class of all sets, as though all sets were made available to us by unbounded quantification. So, what (if any) is the fact of the matter?

Debates over the nature of the infinite, whether it be actual or potential or both, date already to antiquity. The two viewpoints we have summarised above extend this kind of debate to the nature of one specific instance of the infinite, the universe of all sets. Potentialists believe that this object is neither actual nor actualisable, whereas actualists do. ${ }^{7}$

Now, [MaxExt] seems to fit best the potentialist viewpoint, insofar as it posits the existence of extensions of $V$. For, if $V$ is an actualised domain, how could it possibly be extended?

Therefore, on the potentialist viewpoint as befitting [MaxExt], one cannot even speak of $V$, as there is no such actualised and determinate object as $V$, but one should rather refer to an endless sequence of initial segments $V_{\alpha}$ 's whose union can always be extended. This means that [MaxExt] implies, at least, that the cumulative hierarchy is open-ended and that new stages in the formation of sets can always be formed. However, as we have seen, this is just one way of extending the universe. Extensions of $V$ are not only extensions of its height, but also of its width. The width of the universe is given by the power-set operation and, thus, an extension of $V$ in width means that there is also a way to expand the range of the power-set operation.

So, now we have a more complex picture. One could be either an actualist or a potentialist in either height or width, as summarised below:

Height actualism: the height of $V$ is fixed, that is, no new ordinals can be added, WIDTH ACTUALISM: the width of $V$ is fixed, that is no new subsets can be added.

Height Potentialism: the height of $V$ is not fixed, new ordinals can always be added, WidTh POTENTIALisM: the width of $V$ is not fixed, new subsets can always be added.

and combinations thereof.

As said, [MaxExt] seems to commit us to a full-blown form of potentialism, both in height and width. This makes full sense, especially from the point of view of 'quasicombinatorialism': by this attitude, no internal limitation of the procedures to form new sets should be applicable and this also extends to such large-scale objects as $V$.

However, there is one difficulty with this view. While height potentialism seems to be robustly supported by our idea of 'adding' new ordinal-indexed stages to the cumulative

\footnotetext{
${ }^{7}$ An examination of the potentialist and actualist positions, with reference to the justification of reflection principles, is carried out in Koellner (2009), which also draws upon Tait (1998a) and Tait (1998b). A thoroughly actualist point of view on reflection is expounded in Horsten and Welch (2013). A potentialist conception is described in Linnebo (2013), which provides a modal account of the axioms of set theory already explored in Hellman (1989) and Parsons (1983). For the early debate on such issues as the nature of the universe of sets, the role of the absolute infinite and proper classes, all of which are relevant to the actualism/potentialism debate, also see the indispensable Hallett (1984), as well as Jané (1995), and Wang (1996), which contains Gödel's late conceptions on $V$.
} 
hierarchy, so that we can always form a sequence of $V_{\alpha}$ 's increasing with $\alpha$, it is far more problematic to see how extensions of the width of the universe may come in 'stages'. In fact, such extensions as, for instance, the possible set-generic extensions of the universe are not organised in stages at all.

Therefore, whereas our intuitions about the [MIC] seem to suggest that the universe is a fully potential hierarchy of sets, in both height and width, it could be argued that it is simply not possible to make sense of extensions of the universe in width in a way which is in line with the iterative, stage-like character of the [MIC].

The Hyperuniverse Programme has recently fostered a conception which acknowledges the significance of this objection, ${ }^{8}$ and that, therefore, follows a conception alternative to full-blown potentialism which historically was first brought forward by Zermelo. We now proceed to briefly review Zermelo's conception.

3.2. Zermelo's account: a 'vertical' multiverse. As is known, in his seminal paper Zermelo (1930), Zermelo investigates 'natural models' of his axioms, that is, models indexed by boundary numbers (fixed ordinals). Zermelo also proves that natural models form a linear hierarchy by inclusion. An example of a natural model of ZFC is given by $V_{\kappa}$, where $\kappa$ is a strongly inaccessible cardinal.

Now, as said, we construe Zermelo's position as a specific one in the actualism vs potentialism debate: the Zermelian account is potentialist in height and actualist in width.

Zermelo's actualism in width follows from the presence of second-order quantifiers in (some of) his axioms. In fact, Zermelo's 1930 axiomatisation is, essentially, second-order. It is this fact that allows him to establish the quasi-categoricity of set theory or, in more rigorous terms, that:

Theorem 1. Given any two extensional and well-founded structures $M_{1}$ and $M_{2}$, such that $M_{1} \models Z_{2}$ and $M_{2} \models Z_{2}$ (where $Z_{2}$ denotes the axioms of second-order set theory), only three cases can occur: $M_{1}$ is isomorphic to $M_{2}, M_{1}$ is isomorphic to a proper initial segment of $M_{2}$, or $M_{2}$ is isomorphic to a proper initial segment of $M_{1}$.

A trivial consequence of quasi-categoricity is the absoluteness of the power-set operation, which automatically leads one to width actualism. However, our emphasis, here, is on the 'quasi-' bit of his result, since models may still differ in height and, thus, be extendible in a way which clearly suggests height potentialism. In particular, Zermelo construed the sequence of $V_{\alpha}$ 's as stopping points in an endless process of potentialisation of an only temporarily actualised universe.

Zermelo vividly recapitulates his approach in the following manner:

To the unbounded series of Cantor's ordinals there corresponds a similarly unbounded double-series of essentially different set-theoretic models, in each of which the whole classical theory is expressed. The two polar opposite tendencies of the thinking spirit, the idea of creative advance and that of collection and completion [Abschluss], ideas which also lie behind the Kantian 'antinomies', find their

${ }^{8}$ See, in particular, Antos et al. (2015) and Friedman (nd). 
symbolic representation and their symbolic reconciliation in the transfinite number series based on the concept of well-ordering. This series reaches no true completion in its unrestricted advance, but possesses only relative stopping-points, just those 'boundary numbers' [Grenzzahlen] which separate the higher model types from the lower. Thus the set-theoretic 'antinomies', when correctly understood, do not lead to a cramping and mutilation of mathematical science, but rather to an, as yet, unsurveyable unfolding and enriching of that science. (Zermelo (1930), in Ewald (1996), p. 1233)

Zermelo's sequence of natural models can also be viewed as a tower-like multiverse, a 'vertical' multiverse, a collection of universes linearly ordered by inclusion.

Unfortunately, at the practical level, the 'vertical' multiverse fits only half of [MaxExt]: extensions in height are now incorporated within this picture, whereas extensions in width are banned. However, as we said, this seems to be more in line with some worries concerning the impossibility, from a mathematical point of view, to account for extensions in width in an orderly fashion.

Therefore, if we want to keep full potentialism and Zermelo's account, we have to find a way to address also extensions in width within this account. This task we accomplish in the second half of the next section, by introducing $V$-logic.

\section{The hyperuniverse (HI). $V$-LOGiC}

In the previous sections we have established two facts: 1) intrinsic evidence relates to the $[\mathrm{MIC}]$, in particular, to one of its features, that is [MaxExt]; 2) as we have seen, [MaxExt] seems to be more in line with a full-blown potentialist picture of the universe. However, there is no way to address extensions of the width of the universe in a way which suits the iterative character of the [MIC], therefore we ought to settle on an account of $V$ wherein the width of the universe is fixed. Such an account is very fittingly provided by the Zermelian 'vertical' multiverse.

Before turning to the programme's maximality principles in the next section, we first have to carry out two tasks: we have to show that there is indeed a way to formulate principles addressing extensions of the universe not only in height but also in width within a Zermelian conceptual framework and, secondly, we have to identify universes where firstorder consequences of such principles hold. We start with the latter goal: the hyperuniverse provides an ontological environment where one can investigate consequences of our maximality principles.

4.1. The hyperuniverse. Let us leave aside, for a moment, the concept of set, the [MIC] which constitutes its full expression, the ensuing picture of the realm of sets as the cumulative hierarchy and let us turn our attention to the techniques used by set-theorists to establish results concerning set-theoretic truth.

As is known, there is only one way to establish the independence of set-theoretic statements from the axioms, i.e. through finding two models wherein that statement and its 
negation are, respectively, true. If the axioms are consistent, then they cannot prove or disprove such a statement.

There is a wide variety of models that set-theorists investigate: e.g., the constructible universe $L$, core models $K, H O D, M[G]$ (where $G$ is a generic filter on a forcing poset $\mathbb{P} \in M)$ and so forth. The main techniques employed consist in the construction of an inner model and of a forcing extension of a ground model $M$. Almost invariably, the ground model used is a countable transitive model.

So, the problem is the following: how do all these models relate to the concept of set, which seemed to give rise to a unique picture of the realm of sets, that is, $V$ ? Moreover, does each of these models constitute a separate and, to some extent, alternative ontological construct?

The situation we are to face up to here is direly ambivalent. On the one hand, one could legitimately claim that all model-theoretic constructions are in $V$, 'reflecting' the universe each in its own particular way. On the other hand, one could say that, if $V$ is a fully determinate construct, something which seems plausible in light of our adoption of the [MIC] and of its associated Bernaysian 'quasi-combinatorialism', then all of these models represent different and, sometimes, mutually incompatible versions of set-theoretic truth, which cannot possibly be amalgamated into one single framework.

Now, call the view that there is a single universe of sets monism, whereas let pluralism be the view that there are many universes, and that $V$ has no ontological priority. Our approach is alternative to both and may be legitimately called 'dualistic'. Within the programme, we are, in a sense, forced to postulate both the existence of one 'extendible' universe and, at the same time, that of a plural framework containing many universes, where properties of the universe allow the detection of further set-theoretic truth. Now, the models we want to confine our attention to are countable transitive models and our plural framework is defined as follows:

Definition 1 (Hyperuniverse). Let $\mathbb{H}^{Z F C}$ be the collection of all countable transitive models of $Z F C$. We call $\mathbb{H}^{Z F C}$ the hyperuniverse. ${ }^{9}$

But just why should one confine one's attention only to countable transitive models? Our choice is not related to the concept of set and rather originates from concerns arising from practice: we want to infer new truth (first-order statements) from intrinsically motivated new axioms (maximality principles) and, in order to do this, countable transitive models are not only suitable, but also necessary (more details on this are given below in our discussion of $V$-logic). Further reasons for adopting the hyperuniverse as a multiverse construct are more precisely substantiated in what follows:

(1) First of all, it should be noticed that $\mathbb{H}$ is closed under forcing and inner models, which, as we saw, are the main techniques in the current practice. In other terms, if we start with countable transitive models, the use of forcing and inner models does not require more than and leave us with countable transitive models.

\footnotetext{
${ }^{9}$ Henceforth, we shall only use $\mathbb{H}$ to refer to it.
} 
(2) The satisfaction of maximality principles in countable transitive models is also already suggested by the Löwenheim-Skolem theorem: given a statement $\phi$, if $\phi$ is true in $V$, then $\phi$ is true in some element of the hyperuniverse. However, the notion of 'satisfaction', here, has to be mathematically secured more robustly (see section $\S 4.2$ below).

(3) In $\mathbb{H}$, as a consequence of its very definition, there is no ill-founded model, and this fact is perfectly in line with our motivating evidential framework, that is, the [MIC].

Therefore, the adoption of the hyperuniverse is entirely subservient to achieving the result we wish to attain, that of finding new set-theoretic truth, but, as we have seen, is also well justified in light of different concurrent considerations and, in particular, of the fact that countable transitive models constitute the main tool used by set-theorists to investigate settheoretic truth, a tool whereby the iterative and well-founded character of the cumulative hierarchy expressed by the [MIC] can be very aptly reproduced in a small-scale context.

4.2. $V$-logic. We now proceed to describe how one can make sense of width maximality using $V$-logic. Such width maximality principles include the IMH, SIMH, IMH\# and SIMH\#, all of which will be defined in the next section.

As we said, the Löwenheim-Skolem theorem allows one to argue that any first-order property of $V$ reflects to a countable transitive model. However, on a closer look, one needs to deal with the problem that not all relevant properties of $V$ are first-order over $V$. In particular, the property of $V$ 'having an outer model (a 'thickening') with some first-order property' is a higher-order property. We show now that, with a little care, all reasonable properties of $V$ formulated with reference to outer models are actually first-order over a slight extension ('lengthening') of $V$.

We first have to introduce some basic notions regarding the infinitary logic $L_{\kappa, \omega}$, where $\kappa$ is a regular cardinal. ${ }^{10}$ For our purposes, the language is composed of $\kappa$-many variables, up to $\kappa$-many constants, symbols $\{=, \in\}$, and auxiliary symbols. Formulas in $L_{\kappa, \omega}$ are defined by induction: (i) All first-order formulas are in $L_{\kappa, \omega}$; (ii) Whenever $\{\varphi\}_{i<\mu}, \mu<\kappa$ is a system of formulas in $L_{\kappa, \omega}$ such that there are only finitely many free variables in these formulas taken together, then the infinite conjunction $\bigwedge_{i<\mu} \varphi_{i}$ and the infinite disjunction $\bigvee_{i<\mu} \varphi_{i}$ are formulas in $L_{\kappa, \omega}$; (iii) if $\varphi$ is in $L_{\kappa, \omega}$, then its negation and its universal closure are in $L_{\kappa, \omega}$. Barwise developed the notion of proof for $L_{\kappa, \omega}$, and showed that this syntax is complete, when $\kappa=\omega_{1}$, with respect to the semantics (see discussion below and Theorem 2).

Let us now consider a special case of $L_{\kappa, \omega}$, the so-called $V$-logic. Suppose $V$ is a transitive set of size $\kappa$. Consider the logic $L_{\kappa^{+}, \omega}$, augmented by $\kappa$-many constants $\left\{\bar{a}_{i}\right\}_{i<\kappa}$ for all the elements $a_{i}$ in $V$. In this logic, one can write a single infinitary sentence which ensures that if $M$ is a model of this sentence (which is set up to ensure some desirable property of $M$ ), then $M$ is an outer model of $V$ (satisfying that desirable property). Now, the crucial point

\footnotetext{
${ }^{10}$ Full mathematical details are in Barwise (1975). We wish to stress that the infinitary logic discussed in this section appears only at the level of theory as a tool for discussing outer models. The ambient axioms of ZFC are still formulated in the usual first-order language.
} 
is the following: if $V$ is countable, and this sentence is consistent in the sense of Barwise, then such an $M$ really exists in the ambient universe. ${ }^{11}$ However, if $V$ is uncountable, the model itself may not exist in the ambient universe, but, in that case, we still have the option of staying with the syntactical notion of a consistent sentence. ${ }^{12}$

We have to introduce one further ingredient, that of an admissible set. $M$ is an admissible set if it models some very weak fragment of ZFC, called Kripke-Platek set theory, KP. What is important for us here is that for any set $N$, there is a smallest admissible set $M$ which contains $N$ as an element $-M$ is of the form $L_{\alpha}(N)$ for the least $\alpha$ such that $M$ satisfies KP. We denote this $M$ as $\operatorname{Hyp}(N)$.

And we have the following crucial result:

Theorem 2 (Barwise). Let $V$ be a transitive set model of ZFC. Let $T \in V$ be a firstorder theory extending ZFC. Then there is an infinitary sentence $\varphi_{T, V}$ in $V$-logic such that following are equivalent:

(1) $\varphi_{T, V}$ is consistent.

(2) $\operatorname{Hyp}(V) \models " \varphi_{T, V}$ is consistent."

(3) If $V$ is countable, then there is an outer model $M$ of $V$ which satisfies $T$.

By Theorem 2, if we wish to talk about outer models of $V$ ('thickenings', that is, extensions of the width of $V$ ), we can do it in $\operatorname{Hyp}(V)$ - a slight lengthening of $V$ - by means of theories, without really thickening our $V$, that is, without postulating that such extensions are real. However, if we wish to have models of the resulting consistent theories, then, using the Löwenheim-Skolem theorem, we can shift to countable transitive models. And this is precisely where the hyperuniverse comes into play.

Now, we also want to make sure that members of the hyperuniverse really witness statements expressing the width maximality of $V$. One such statement is the Inner Model Hypothesis or IMH (for whose full examination see next section).

$V$ satisfies the IMH if for every first-order sentence $\psi$, if $\psi$ is satisfied in some outer model $W$ of $V$, then there is a definable inner model $V^{\prime} \subseteq V$ satisfying $\psi$. The formulation of IMH requires the reference to all outer models of $V$, but with the use of infinitary logic, we can formulate IMH syntactically in $\operatorname{Hyp}(V)$ as follows: $V$ satisfies IMH if for every $T=Z F C+\psi$, if $\varphi_{T, V}$ from Theorem 2 above is consistent in $\operatorname{Hyp}(V)$, then there is an inner model of $V$ which satisfies $T$. Finally, with an application of the Löwenheim-Skolem theorem to $\operatorname{Hyp}(V)$, this becomes a statement about elements of the hyperuniverse.

\section{MAXimality PRINCIPLES FOR $V$}

We have now arrived at the crux of our paper. Within the programme, we cast our new axioms as maximality principles about $V$ and, after having established, using the notion of satisfaction in $V$-logic, that 1) these principles can be formulated in a Zermelian

\footnotetext{
${ }^{11}$ Again, for more details we refer the reader to Barwise (1975).

${ }^{12}$ This means that the hyperuniverse, although fully justifiable in view of the use of $V$-logic, can be disposed of, if one only wants to keep the Zermelian multiverse (and its immediate connection with the $[\mathrm{MIC}]$ and $[\mathrm{MaxExt}])$.
} 
framework and 2) they are satisfied by members of $\mathbb{H}$, we can also see what first-order consequences they have through the study of countable transitive models, i.e. elements of the hyperuniverse.

First, there is one point which should be emphasised again: as the reader will see in a moment, the maximality principles that have been formulated within the programme all address extensions of $V$ and, therefore, in our view, they specify ways such extensions, as postulated by [MaxExt], should be conceived of. Thus all such principles can be seen as specifications of [MaxExt]. As our evidential framework for the search for new axioms was given by the $[\mathrm{MIC}]$ and these principles follow from this evidential framework quite naturally, we believe that we have in this way found a source for new axioms based on the maximal interative conception.

Predictably, some principles refer to extensions in height and others to extensions in width. Accordingly, we may say that the former address the vertical maximality and the latter the horizontal maximality of the universe.

Vertical maximality has been recently formulated by the first author and Honzik in terms of a strong form of reflection called \#-generation. We do not discuss the details here, but refer the reader to their paper Friedman and Honzik (nd).

Let us instead examine horizontal maximality. In the programme, this property is expressed by the IMH.

Definition 2 (IMH). If for every first-order sentence $\psi$, if $\psi$ is satisfied in some outer model $W$ of $V$, then there is a definable inner model $V^{\prime} \subseteq V$ satisfying $\psi$.

Just to make things as clear as possible, 'outer models', in the definition above, are precisely the formal equivalent of extensions of the universe in width. Moreover, in our view, IMH prescribes the maximality of the universe (by using the language of 'extensions'), insofar as it prescribes its maximality with respect to inner models. Universes satisfying the IMH exist in $\mathbb{H}$ :

Theorem 3. Assuming the consistency of large cardinals, there are members of the hyperuniverse which satisfy the IMH.

The proof is in Friedman et al. (2008), where it is shown that the consistency of slightly more than the existence of a Woodin cardinal is sufficient. One might question the use of Woodin cardinals here, which may not be intrinsically justified. But note that it is not the existence of Woodin cardinals that is needed to obtain the existence of members of $\mathbb{H}$ satisfying the IMH. It is only the consistency of Woodin cardinals that is used as an auxiliary mathematical tool in order to construct universes satisfying IMH and we believe that this fact does not commit us to asserting the existence of such cardinals, as 'consistency' is far less than 'existence'. It should be noted, incidentally, that in all members of $\mathbb{H}$ satisfying IMH there are no large cardinals at all. Therefore, if one believes that IMH is a correct higher-order principle about $V$, then one obtains that there are no large cardinals in $V$. But the IMH does not take vertical maximality into account. Let IMH\# denote the IMH for vertically-maximal, i.e. for \#-generated, universes. In other words, $M$ satisfies the 
IMH\# if $M$ is \#-generated and whenever a first-order sentence holds in a \#-generated outer model of $M$, it also holds in a definable inner model of $M$.

Theorem 4. There are members of the hyperuniverse which satisfy IMH\#.

For a proof see Friedman and Honzik (nd). The attraction of IMH\# is that it captures aspects of both vertical and horizontal maximality simultaneously.

We also mention some strengthenings of the principles given above. An absolute parameter is a set $p$ which is uniformly definable over all outer models of $V$ which 'respect $p$ ', i.e. which preserve cardinals up to and including the cardinality of the transitive closure of $p$. The SIMH (Strong IMH) is the IMH for sentences with absolute parameters relative to outer models which respect them: if a sentence with absolute parameters holds in an outer model which respects those parameters then it holds in a definable inner model.

A related principle is the CPIMH (Cardinal Preserving IMH). A cardinal-absolute parameter is a set $p$ which is uniformly definable over all cardinal-preserving extensions of $V$. Then CPIMH asserts that if a sentence with cardinal-preserving parameters holds in a cardinal-preserving outer model of $V$ it also holds in a definable inner model of $V$.

Restricting to \#-generated universes yields corresponding principles SIMH\# and CPIMH\#.

We do not know whether there are elements of $\mathbb{H}$ satisfying SIMH, CPIMH or their \#-versions, but it is reasonable to conjecture that they do. ${ }^{13}$ We have:

Theorem 5. (see Friedman (2006))

(a) In all universes satisfying IMH, PD is false, and there are no large cardinals.

(b) All universes which satisfy SIMH, CPIMH or their \#-versions also satisfy $\neg C H$.

Thus maximality principles emanating from the Hyperuniverse Programme do indeed have striking first-order consequences.

\section{NEW AXIOMS AS $\mathbb{H}$-AXIOMS}

6.1. The nature of $\mathbb{H}$-axioms. As we said at the beginning, we do not want to advocate any specific first-order new axiom in this paper, but rather present an alternative conceptual framework whereby higher-order statements are indeed new axioms, which also happen to have important first-order consequences. The framework we have presented, in particular the mathematical results detailed in the previous section, lend support to the following conclusion: members of $\mathbb{H}$ satisfying maximality principles have remarkable properties, e.g. in all countable transitive models satisfying IMH, PD is false and in all of them satisfying $\mathrm{CPIMH} \#, \mathrm{CH}$ is false.

Now, let us focus our attention for a moment on $\neg \mathrm{PD}$ and $\neg \mathrm{CH}$. These first-order settheoretic statements are consequences of new axioms that:

(1) hold in 'local' areas of $\mathbb{H}$

(2) are expressed in terms of intrinisically motivated maximality principles as, respectively, IMH and CPIMH\#.

\footnotetext{
${ }^{13}$ In particular, there are universes which obey them restricted to the parameter $\omega_{1}$.
} 
By virtue of this, we label IMH and CPIMH\# $\mathbb{H}$-axioms, insofar as they hold in specific portions of $\mathbb{H}$ and are intrinsically motivated on the grounds of the [MIC] and [MaxExt].

Again, it is important to emphasise on what grounds our claim can be made: using $V$-logic, we can characterise the relationship between maximality principles and their consequences as mirroring that between higher-order properties of $V$ and first-order truths in members of $\mathbb{H}$. In particular, in the Hyperuniverse Programme higher-order properties of $V$ are, in a sense, turned into $\mathbb{H}$-axioms, properties of members of $\mathbb{H}$ expressible through (first-order) quantification over $\mathbb{H}$.

Furthermore, we also claim that $\neg \mathrm{PD}$ would be, in accordance with our conceptual presuppositions, an intrinsically motivated new set-theoretic truth insofar as IMH is an intrinsically motivated maximality principle.

Of course there are members of $\mathbb{H}$ which do not satisfy the IMH. Consequently, $\neg$ PD is a statement holding only in a portion of $\mathbb{H}$, something which accounts for our idea that $\mathbb{H}$-axioms are 'local' axioms. This is inevitable if one wishes to be conceptually faithful to the multiverse phenomenon.

However, there is a global corrective to this 'pluralistic' view. The programme strives for the identification of an 'optimal' maximality principle ( $\mathbb{H}$-axiom). Now, suppose that $P$ were such a principle; we would then exclude any member of $\mathbb{H}$ which would not satisfy $P$ and therefore $P$ could be taken to be the 'new' $\mathbb{H}$-axiom we are searching for, derivable from the maximal iterative conception and with intrinsically justified first-order consequences.

It could be objected that viewing axioms as 'consequences' of more general principles implies that one accepts these 'consequences' without understanding their 'content', in particular whether they are 'intuitively true' and this would distance our methodology from a genuine search for 'meaningful' additions to ZFC. However, the methodology envisaged here precisely aims to provide an alternative notion of 'intuitively true' as based on the acceptance of the intuitive truth of maximality principles concerning $V$. Therefore, in our view, the 'meaningfulness' of the consequences of a maximality principle is guaranteed by the meaningfulness of the principle itself.

6.2. Alternative approaches. Finally, we go back, again, to the issue we started with at the beginning of this paper: what new axioms should be. First of all, we will try to dispel one main worry about the methodology described, namely, that it could imply that all new axiom candidates other than $\mathbb{H}$-axioms should automatically fail to be viewed as plausible new axioms and, what is worse, as lacking any evidence in favour of their acceptance.

This would be a gross misrepresentation of our perspective. In the previous subsection, when we regarded $\neg \mathrm{PD}$ or $\neg \mathrm{CH}$ as consequences of new axioms, our aim was not to make a general argument in favour of the rejection of $\mathrm{PD}$ or $\mathrm{CH}$. At the same time, nowhere in this paper have we suggested that the 'current' new axioms should all be rejected: the proof of this is that, again, $\mathrm{PD}, \mathrm{CH}$ or their negations have already been subjected to extensive mathematical investigations as new axioms, and, in this respect, our programme has nothing new to add.

What we have tried to establish here is that, if our evidential framework is preferable to others, then there are reasons to think that PD might be rejected precisely on its grounds. 
Leaving aside our framework for a moment, it is maybe appropriate to make a brief digression on the status of PD. Over the years, PD has been celebrated as a new axiom for which there is a significant body of evidence. ${ }^{14}$ In particular, two aspects are almost invariably highlighted: 1) PD is successful, because it makes the theory of sets of reals up to and including the projective sets behave well (under PD, all projective sets of reals are Lebesgue measurable and every uncountable projective set of reals has a perfect subset, which means that $\mathrm{CH}$ cannot be projectively refuted); 2) PD remarkably connects two apparently distant areas of set theory, descriptive set theory and the theory of large cardinals, as it was proved that the existence of Woodin cardinals and PD have the same consistency strength.

However, arguments in favour of PD are mostly extrinsic and are based on the fact that it follows from large cardinals or from set-generic absoluteness principles, but justifications are lacking for both the existence of large cardinals and for a form of absoluteness which imposes an artificial restriction to set-forcings. ${ }^{15}$ Also, advocates of PD often claim that truth is taken to be based solely on current set-theoretic practice, ignoring what is relevant for mathematics outside of set theory or for the maximal iterative conception. So arguing that PD can be inferred from current set-theoretic practice may be insufficient for claiming its truth. ${ }^{16}$

Now, returning to our main topic, why should all other proposed definitions of what a 'new axiom' should be like be replaced by ours? Because other approaches may be fraught with insurmountable difficulties. For instance, consider the following three alternatives:

A new axiom should be a first-order statement true of the concept of set. As we have seen, true of the concept of set means true of the [MIC], but there might be quite a few settheoretic statements for which such criterion cannot apply. For instance, is the Axiom of Choice true of the [MIC]? How about the Axiom of Determinacy? Even if such criterion is applicable, there might be cases where one intrinsically motivated first-order axiom may contradict another enjoying the same status.

\footnotetext{
${ }^{14}$ For the full case for axioms of definable determinacy, such as PD, see, e.g., Woodin (2001), Martin (1998), Koellner (2006).

${ }^{15}$ On this, see $\S 8$ of the present paper.

${ }^{16}$ To be fair, advocacy of PD along an alternative, intrinsic-evidence-based line of thought, has also been made. See, for instance, Hauser (2002): 'But aside from extrinsic evidence, there are other reasons to regard $P D$ as the correct axiom for the projective sets. With the progress made in the theory of canonical models for large cardinals it has become clear that $P D$ is implied by and is in fact equivalent to a vast number of prima facie unrelated combinatorial principles including large-cardinal axioms. Still this may not establish their intrinsic necessity because the relevant large-cardinal axioms at present do not enjoy the same kind of intrinsic plausibility as for example Mahlo cardinals. However, the intrinsic necessity of an axiom need not be immediate and could depend on the discovery of additional facts' (p. 274). Of course, at present it is not clear what 'intrinsic' facts would add to the defensibility of PD and whether they will ultimately be discovered.
} 
A new axiom should be a first-order statement, not intrinsically, but rather extrinsically justified. Many new axioms such as forcing axioms or PD and, in general, definable determinacy axioms, have a lot of strong extrinsic support. However, this fact may not be sufficient and, in fact, too limited. For instance, in the Appendix ( $\S 8$ ), we present arguments showing that extrinsically supported absoluteness axioms may be inadequate.

A new axiom is an axiom which is 'practically' confirmed, that is, verified empirically in specific areas of set theory'. This is a refinement of the statement above. However, the definition is still problematic, as the notion of an axiom's being 'practically confirmed' is obscure and would require clarification. ${ }^{17}$

We do not know whether the above procedure to identify and justify $\mathbb{H}$-axioms and the notion of $\mathbb{H}$-axiom itself will become standard. It does seem to us that our proposal responds better to the conceptual difficulties of the aforementioned alternative approaches. In particular, after the substantial demise of 'Gödel's programme', the search for new intrinsically motivated new axioms is at a loss within all other current research programmes. The reasons have been amply considered above, especially in our introductory remarks: the notion of set-theoretic truth falls short of a unique characterisation, if it is to reflect a unique realm of objects, in particular as a consequence of the existence of the multiverse, and it does not seem that this situation can be easily repaired, unless one adopts higher-order principles motivated by the concept of set.

\section{Concluding Summary}

In this paper, we have shown how the search for new axioms is carried out within the Hyperuniverse Programme. The methodology devised is motivated by the existence of three concurrent phenomena: 1) the set-theoretic multiverse; 2) the availability of higher-order principles describing forms of maximality of $V$ in line with the [MIC], that is, $\mathbb{H}$-axioms; 3 ) a demonstrable link between such maximality principles and countable transitive models.

Maximality principles, that specify different notions of the maximality of $V$, also have, through the use of $V$-logic, robust consequences in countable transitive models. Obviously, different maximality principles may have different first-order consequences. So, the main shortcoming of this conception is that it is not sufficient to fix set-theoretic indeterminacy uniquely. However, we believe that the further development of the programme may establish the existence of an 'optimal' maximality criterion, which, in turn, may lead to the acceptance of one single, intrinsically justified collection of first-order statements to be added to ZFC.

The project is open to further generalisations and developments. New maximality principles will come out, helping us to identify further universes where certain set-theoretic

\footnotetext{
${ }^{17}$ For further details on these different approaches, see, respectively: 1) on the strength and value of extrinsic justifications, Maddy (1996), Maddy (1997), Koellner (2006), Martin (1998); 2) on second-order logic and set theory, Shapiro (1991), and Jané (2005a); 3) on the quasi-empirical view, again, Koellner (2006), or Hauser (2002).
} 
statements do or do not hold. A more careful description of $V$, of different types of universes in $\mathbb{H}$, and axioms therein, may, therefore, be on its way.

\section{Appendix: Absoluteness Axioms}

In recent years, considerable attention has been paid by set-theorists to what we may call the absoluteness programme. The main goal of this programme is to foster suitable mathematical strategies and principles (absoluteness axioms) to 'induce' the absoluteness of certain set-theoretic statements across an appropriately selected collection of models (or set-theoretic multiverse). ${ }^{18}$

Although absoluteness axioms have received a lot of extrinsic support in recent years, ${ }^{19}$ here we want to present evidence that no new first-order absoluteness axiom has good prospects to be viewed as a plausible axiom candidate extending ZFC. As far as the 'extrinsic' value of these axioms is concerned, the reasons for this claim are structural, that is, refer to internal features of the absoluteness phenomenon and do not depend upon the nature and the content of the axiom under consideration.

We now explain why this is so.

Recall the Lévy hierarchy of logical formulas: one starts with $\Delta_{0}$-sentences, those with only bounded quantifiers. $\Sigma_{1}$ - and $\Pi_{1}$ - sentences contain, respectively, one block of existential or one block of universal quantifiers followed by bounded quantifiers and, in general, $\Sigma_{k+1}=\exists x_{1} x_{2} \ldots x_{n} \Pi_{k}$ and $\Pi_{k+1}=\forall x_{1} x_{2} \ldots x_{n} \Sigma_{k}$. Also, recall that $H(\kappa)$ denotes the union of all transitive sets of size less than $\kappa .^{20}$ The $\Sigma_{n}$-theory of $H(\kappa)$ is the set of $\Sigma_{n}$-sentences true in $H(\kappa)$.

Definition 3. We say that $M \sqsubseteq N$ if $M \subseteq N$ are transitive models of $Z F C$ with the same ordinals.

Now, there exist trivial forms of absoluteness. For instance, as is known, if $M \sqsubseteq N$, where $M$ and $N$ are models of ZFC, the theory of $H(\omega)$ is the same in $M$ and $N$. Going one level higher in the hierarchy of $H(\kappa)$, one finds the following seminal result due to Lévy and Shoenfield:

Theorem 6. If $M \sqsubseteq N$ are models of $Z F C$, then the $\Sigma_{1}$-theory of $H\left(\omega_{1}\right)$ is the same in $M$ and $N$.

Now, what about the $\Sigma_{2}$-theory of $H\left(\omega_{1}\right)$ ? Climbing up the scale of complexity of settheoretic sentences, absoluteness comes to a halt:

Theorem 7. There are models $M \sqsubseteq N$ of ZFC such that the $\Sigma_{2}$-theory of $H\left(\omega_{1}\right)$ is not the same in $M$ and $N$.

\footnotetext{
${ }^{18}$ Given a formula $\phi$ and transitive models $M$ and $N$, we say that $\phi$ is absolute between $M$ and $N$ iff $\phi^{M}\left(x_{1}, x_{2}, \cdots, x_{n}\right) \leftrightarrow \phi^{N}\left(x_{1}, x_{2}, \cdots, x_{n}\right)$.

${ }^{19}$ For an introductory overview of some of these see Bagaria (2000).

${ }^{20}$ That is, the union of all sets whose transitive closure has cardinality less then $\kappa$.
} 
Proof. The statement "there is a nonconstructible real" is a $\Sigma_{2}$ property of $H\left(\omega_{1}\right)$. Take $N$ to satisfy this and $M$ to be $L^{N}$.

This negative result may be circumvented via a two-step strategy: the first step consists in restricting the $\sqsubseteq$-relation in a suitable way. Consider the following definition:

Definition 4. $M \sqsubseteq^{\text {set-generic }} N$ iff $N$ is a set-generic extension of $M$.

Theorem 8 (Bukovsky $\left.{ }^{21}\right)$. $M \sqsubseteq$ set-generic $N$ iff $M \sqsubseteq N$ and for some cardinal $\kappa$ of $M$ every function in $N$ on a set in $M$ into $M$ is contained in a multi-valued function in $M$ with fewer than $\kappa$ values for each argument.

One further refinement of this definition leads to the following notion:

Definition 5. $M \sqsubseteq$ stationary-preserving-set-generic $N$ iff $N$ is a set-generic extension of $M$ and any subset of $\bar{\omega}_{1}^{M}$ which is stationary in $M$ is also stationary in $N$.

In other terms, by restricting the $\sqsubseteq$-relation to, respectively, $\sqsubseteq^{\text {set-generic }}$ or, on the other hand, $\sqsubseteq^{\text {stationary-preserving-set-generic }}$ one only takes into account generic extensions of models obtained through set-forcing or stationary-preserving set-forcing.

The second step in the strategy consists in considering certain extensions of ZFC, say, $\mathrm{ZFC}+\mathrm{Ax}$., and then replacing the multiverse $\mathbb{M}^{Z F C}$ by the multiverse $\mathbb{M}^{Z F C+A x \text {. associ- }}$ ated to the stronger system $\mathrm{ZFC}+\mathrm{Ax}$.

Using this two-step strategy, Woodin and Viale have obtained results which are, no doubt, of mathematical significance, ${ }^{22}$ but, with respect to our foundational project, their results present some crucial shortcomings: 1) the axioms they consider, such as the existence of class-many Woodin cardinals, are not justified intrinsically; 2) the restriction of the -relation to set-generic extensions is unwarranted in view of our definition of $\mathbb{H}$. Furthermore, even leaving these issues aside, it is not clear how far the programme they have been carrying out can be extended, and with what results. We will come to this in a moment.

Alternatively, one could employ only the second step of the above strategy, by supporting the acceptance of axioms such as $V=L$. Gödel's work yields:

Theorem 11. If $M \sqsubseteq N$ are models of $Z F C+V=L$, then $M=N$.

However, promising though this strategy may seem, it reveals the same shortcoming as before, insofar as it is hinged upon the acceptance of a mathematical principle, $V=L$,

\footnotetext{
${ }^{21}$ See Bukovsky (1973).

${ }^{22}$ See, in particular, Woodin (2001) and Viale (nd). Among other things, Woodin proved the following:
}

Theorem 9. If $M \sqsubseteq^{\text {set-generic }} N$ are models of $Z F C+$ large cardinals $+C H$, then the $\Sigma_{1}$-theory of $H\left(\omega_{2}\right)$ (with parameter $\omega_{1}$ ) is the same in $M$ and $N$.

Viale has recently proved:

Theorem 10. If $M \sqsubseteq$ stationary-preserving-set-generic $N$ are models of $Z F C+$ large cardinals $+M M^{+++}$, then the theory of $H\left(\omega_{2}\right)$ is the same in $M$ and $N$. 
which does not possess a sufficient degree of intrinsic motivation in view of our notion of 'intrinsicness' expounded in $\S 5$.

As said, in fact, there is strong evidence that the second step in the above two-step strategy, that of extending ZFC to a stronger first-order theory to obtain greater absoluteness, is doomed to failure. Consider the following:

Theorem 12. Suppose $T$ is a first-order theory, compatible with the following two statements:

(1) the class $\{\alpha: \alpha$ measurable $\}$ is stationary;

(2) the class $\left\{\alpha: V_{\alpha} \prec_{\Sigma_{\omega}} V\right\}$ is unbounded.

Then, $\Sigma_{2}\left(H\left(\omega_{1}\right)\right)$-absoluteness fails for models of $T$ : there are models $M \sqsubseteq N$ of $T$ such that the $\Sigma_{2}$-theory of $H\left(\omega_{1}\right)^{M} \neq \Sigma_{2}$-theory of $H\left(\omega_{1}\right)^{N}$. If $T$ consists of only a finite set of axioms then (2) above is not needed.

Sketch of Proof. The hypotheses imply that there is a model $V$ of ZFC with a largest measurable $\kappa$ such that $T$ holds in $V_{\kappa}$. Now iterate the measure on $\kappa$ through the ordinals, resulting in a model $N$. In $N$, there is a model $V_{0}$ like $V$ but only satisfying KP, with an iterable top measure. Again iterate the top measure through the ordinals to form an inner model $M$. Then $M \sqsubseteq N$ are both models of $T$ but by choosing $V_{0}$ minimally we can arrange that in $M$ there is no iterable model $P$ of KP with a top measurable $\kappa_{0}$ such that $T$ holds in the $V_{\kappa_{0}}$ of $P$. This $\Pi_{2}\left(H_{\omega_{1}}\right)$ sentence fails in $N$ and this gives the asserted failure of absoluteness.

The theorem asserts that any first-order theory which is compatible with the existence of measurable cardinals (in fact, a stationary class of measurable cardinals) fails to ensure $\Sigma_{2}\left(H\left(\omega_{1}\right)\right)$ absoluteness for its models. This is very strong evidence against the use of firstorder axioms for obtaining convincing absoluteness principles for $\Sigma_{2}\left(H\left(\omega_{1}\right)\right)$ statements.

To summarise, there is a network of results which seem to show that, through the adoption of absoluteness axioms, one can find new set-theoretic truth, by extending the absoluteness of set-theoretic statements to levels of increasing first-order complexity. However, first of all, none of the axioms adopted or used in the programme seems to be intrinsically motivated. Secondly, there is also some evidence that such an extension collides with the existence of measurable cardinals. As a consequence, one appears to be forced to artificial restrictions of the multiverse to only certain models or of the notion of absoluteness itself.

Consequently, we come to the following conclusion: no first-order absoluteness axiom has good prospects of being accepted as a new axiom on the grounds of both intrinsic or extrinsic justifications.

In our opinion, if one wants to spell out a plausible conception of 'truth in the multiverse', one has to proceed in the alternative way we propose, through the use of higher-order principles. 


\section{REFERENCES}

Antos, C., Friedman, S.-D., Honzik, R., and Ternullo, C. (2015). Multiverse Conceptions in Set Theory. Synthese, 192(8):2463-2488.

Arrigoni, T. and Friedman, S. (2012). Foundational Implications of the Inner Model Hypothesis. Annals of Pure and Applied Logic, 163:1360-66.

Arrigoni, T. and Friedman, S. (2013). The Hyperuniverse Program. Bulletin of Symbolic Logic, 19(1):77-96.

Bagaria, J. (2000). Bounded Forcing Axioms and Generic Absoluteness. Archives for Mathematical Logic, 39:393-401.

Barwise, J. (1975). Admissible Sets and Structures. Springer Verlag, Berlin.

Benacerraf, P. and Putnam, H., editors (1983). Philosophy of Mathematics. Selected Readings. Cambridge University Press, Cambridge.

Bernays, P. (1935). Sur le Platonisme dans les Mathématiques. L'Enseignement Mathématique, 34:52-69.

Bukovsky, L. (1973). Characterization of generic extensions of models of set theory. Fundamenta Mathematicae, 83.

Ewald, W., editor (1996). From Kant to Hilbert: A Source Book in the Foundations of Mathematics, volume II. Oxford University Press, Oxford.

Friedman, S. (2006). Internal Consistency and the Inner Model Hypothesis. Bulletin of Symbolic Logic, 12(4):591-600.

Friedman, S. (n.d.). The Philosophy and Mathematics of Set-Theoretic Truth. In Proceedings of the 2014 Chiemsee Summer School 'Proof, Truth and Computation'. IfCoLog Journal of Logics and their Applications.

Friedman, S. and Honzik, R. (n.d.). On Strong Forms of Reflection in Set Theory. Mathematical Logic Quarterly.

Friedman, S., Welch, P., and Woodin, W. H. (2008). On the consistency strength of the Inner Model Hypothesis. Journal of Symbolic Logic, 73(2):391-400.

Gödel, K. (1947). What is Cantor's Continuum Problem? American Mathematical Monthly, 54:515-525.

Hallett, M. (1984). Cantorian Set Theory and Limitation of Size. Clarendon Press, Oxford.

Hauser, K. (2002). Is the Continuum Problem Inherently Vague? Philosophia Mathematica, 10:257-285.

Hellman, G. (1989). Mathematics without Numbers. Towards a Modal-Structural Interpretation. Clarendon Press, Oxford.

Horsten, L. and Welch, P. (2013). Absolute Infinity. Unpublished.

Jané, I. (1995). The role of the Absolute Infinite in Cantor's Conception of Set. Erkenntnis, $42: 375-402$.

Jané, I. (2005a). Higher-order Logic Reconsidered. In Shapiro, S., editor, Oxford Handbook of Philosophy of Mathematics, pages 747-774. Oxford University Press, Oxford.

Jané, I. (2005b). The iterative conception of sets from a Cantorian perspective. In Hájek, P., Westertål, D., and Valdés-Villanueva, L., editors, Logic, Methodology and Philosophy of Science: Proceedings of the Twelfth International Congress, pages 373-393. King's 
College Publications, London.

Koellner, P. (2006). On the question of Absolute Undecidability. Philosophia Mathematica, 14(2):153-188.

Koellner, P. (2009). On Reflection Principles. Annals of Pure and Applied Logic, 157(2$3): 206-19$.

Linnebo, Ø. (2013). The Potential Hierarchy of Sets. Review of Symbolic Logic, 6(2):205228.

Maddy, P. (1988a). Believing the Axioms, I. Bulletin of Symbolic Logic, 53(2):481-511.

Maddy, P. (1988b). Believing the Axioms, II. Bulletin of Symbolic Logic, 53(3):736-764.

Maddy, P. (1996). Set-theoretic Naturalism. Bulletin of Symbolic Logic, 61(2):490-514.

Maddy, P. (1997). Naturalism in Mathematics. Oxford University Press, Oxford.

Martin, D. (1998). Mathematical Evidence. In Dales, H. G. and Oliveri, G., editors, Truth in Mathematics, pages 215-231. Clarendon Press, Oxford.

Parsons, C. (1983). Mathematics in Philosophy. Cornell University Press, Ithaca, NY.

Potter, M. (2004). Set Theory and its Philosophy. Oxford University Press, Oxford.

Shapiro, S. (1991). Foundations without Foundationalism. A Case for Second-Order Logic. Oxford University Press, Oxford.

Tait, W. W. (1998a). Foundations of Set Theory. In Dales, H. G. and Oliveri, G., editors, Truth in Mathematics, pages 273-290. Oxford University Press, Oxford.

Tait, W. W. (1998b). Zermelo on the Concept of Set and Reflection Principles. In Schirn, M., editor, Philosophy of Mathematics Today, pages 469-483. Clarendon Press, Oxford Press, Oxford.

Viale, M. (n.d.). Category forcings, $\mathrm{MM}^{+++}$and generic absoluteness for the theory of strong forcing axioms. Journal of the American Mathematical Society.

Wang, H. (1974). From Mathematics to Philosophy. Routledge \& Kegan Paul, London.

Wang, H. (1996). A Logical Journey. MIT Press, Cambridge (MA).

Woodin, W. H. (2001). The Continuum Hypothesis. Notices of the American Mathematical Society, Part 1: 48, 6, p. 567-76; Part 2: 48, 7, p. 681-90.

Zermelo, E. (1930). Über Grenzzahlen und Mengenbereiche: neue Untersuchungen über die Grundlagen der Mengenlehre. Fundamenta Mathematicae, 16:29-47. 


\section{Contents}

1. New set-theoretic axioms 1

2. Intrinsic evidence for new axioms 3

2.1. Brief remarks on ontology and truth 3

2.2. Two sources of evidence 4

2.3. The maximum iterative concept 5

3. Conceptions of $V$. The 'vertical' multiverse 6

3.1. What is $V$ ? The actualism/potentialism dichotomy 6

3.2. Zermelo's account: a 'vertical' multiverse 8

4. The hyperuniverse $(\mathbb{H})$. $V$-logic $\quad 9$

4.1. The hyperuniverse 9

4.2. $V$-logic 11

5. Maximality principles for $V \quad 12$

6. New axioms as $\mathbb{H}$-axioms 14

6.1. The nature of $\mathbb{H}$-axioms 14

6.2. Alternative approaches $\quad 15$

7. Concluding Summary 17

8. Appendix: Absoluteness Axioms 18

References $\quad 21$ 